\title{
Percepciones sobre convivencia escolar y bullying en una institución educativa de Bogotá
}

\section{Perceptions of school coexistence and bullying in an educational institution in Bogotá}

DOI: http://dx.doi.org/10.17981/cultedusoc.9.1.2018.03

Recibido: 18 de diciembre de 2017/ Aceptado: 25 de marzo de 2018

Lupe García Cano ${ }^{1}$ y Soledad Niño Murcia ${ }^{2}$

Fundación Universitaria Los Libertadores (Colombia)

lgarciac01@libertadores.edu.co

Para citar este artículo

García, L. y Niño, S. (2018). Percepciones sobre convivencia escolar y bullying en una institución educativa de Bogotá. Cultura. Educación y Sociedad 9(1), 45-58. DOI: http://dx.doi.org/10.17981/cultedusoc.9.1.2018.03

\section{Resumen}

Los docentes en su actividad laboral cotidiana se ven enfrentados a situaciones de violencia que no saben cómo enfrentar y que indudablemente repercuten en los ambientes de aprendizaje. El objetivo del estudio fue analizar prácticas pedagógicas para mejorar la convivencia de niñas y niños de $5^{\circ}$ de primaria, las percepciones que estos tienen sobre la convivencia escolar y la existencia de bullying en una institución educativa ubicada en entorno de violencia en Bogotá, Colombia. El método seleccionado fue el estudio mixto, la muestra la conformaron estudiantes, profesores y directivos de un colegio público. Se aplicaron tres instrumentos validados: una encuesta de percepción de convivencia y una encuesta de percepción de bullying aplicadas a estudiantes y una entrevista semi- estructurada aplicada a docentes, coordinadores y directivos. Como resultado, se encontró que se dan las condiciones propicias para que se genere el fenómeno del bullying, sumado a la inexistencia de proyectos pedagógicos que enseñen a los estudiantes a gestionar los conflictos sin recurrir a actos de agresión y de violencia. Se concluye que la institución educativa no reconoce la importancia de la formación valorativa y convivencial desde la primera infancia y prioriza la acumulación de conocimientos, lo cual explica la presencia del conflicto y su manejo violento, conformando un clima hostil para el estudiante.

Palabras Clave: Bullying, convivencia escolar, entornos de violencia, prácticas pedagógicas.

\begin{abstract}
Teachers in their daily work face situations of violence that they do not know how to handle and that undoubtedly impact on learning environments. The objective of the study was to analyze the pedagogical practices to improve the coexistence of girls and boys of 5th grade, the perceptions they have about school coexistence and the need for bullying in an educational institution in Bogotá, Colombia. The selected method was the mixed study, it shows the conformation of students, professors and managers of a public school. Three validated instruments were applied: a survey of perception of coexistence and a survey of perception of bullying applied to students and a semi-structured interview applied to teachers, coordinators and managers. As a result, it was found that there are favorable conditions for the phenomenon of bullying to be generated, coupled with the lack of pedagogical projects that join students in conflicts without resorting to acts of aggression and violence. It is concluded that the educational institution does not recognize the importance of value and coexistence training from early childhood and prioritizes the accumulation of knowledge, which explains the presence of conflict and its violent management, forming a hostile climate for the student.
\end{abstract}

Keywords: Bullying, school coexistence, violence environments, pedagogical practices.

\footnotetext{
${ }^{1}$ Magister en Educación de la Fundación Universitaria Los Libertadores.

${ }^{2}$ Magister en Comunicación de la Fundación Universitaria Los Libertadores.
} 


\section{Introducción}

Este proyecto planteó caracterizar las prácticas pedagógicas utilizadas en la institución educativa de estudio y se identificó la existencia de bullying, así como los sujetos que intervienen, ya sea de manera activa o pasiva. En consecuencia, la investigación planteó como preguntas problema: ¿Qué prácticas pedagógicas, dirigidas a niños, niñas y jóvenes han diseñado los profesores para mejorar la convivencia de instituciones educativas ubicadas en contextos de violencia en el contexto colombiano? ¿Cuál es la percepción de convivencia y del entorno barrial de niños, niñas y jóvenes que estudian en entornos violentos localizados en la cuidad de Bogotá?

El fundamento teórico que sustenta la investigación, está estructurado en tres apartados a saber: bullying, convivencia en la escuela y prácticas pedagógicas.

\section{¿Qué es el bullying?}

Existen varias formas de agresión: el bullying, la agresión física, verbal y social, los ataques sexuales, los encuentros violentos, el comportamiento de pandillerismo y la delincuencia, entre otros (Pepler y Craig, 2000). Pero como lo afirman Ortega, Del Rey y Elipe (2012), no todo conflicto o conducta agresiva puede ser considerado como bullying. En el entorno escolar, cada día se presentan numerosos problemas entre los estudiantes, con un transcurso más o menos complejo y culminan rápidamente con un aprendizaje: "controlar mejor sus deseos y expresar de forma adecuada sus nece- sidades, si quiere lograr la aceptación y el respeto de los demás" (p.207). Lo que sí se sabe, gracias a las investigaciones al respecto, es que muchas de las conductas agresivas terminan siendo bullying.

Según Pepler y Craig (2000), el bullying se diferencia de las demás conductas agresivas, en que hay un desequilibrio de poder entre el victimario o agresor (bully / bullies) y la víctima. Esta última carece de habilidad y competencias sociales para identificar cuándo empieza a ser intimidada, y es incapaz por sí misma de cortar con comportamientos agresivos y abusivos, y mucho menos puede recomponer sus redes afectivas (Ortega, Del Rey y Elipe, 2012).

El bullying se caracteriza por incluir problemas de exclusión social, intimidación, acoso, malos tratos $\mathrm{y}$ también porque es repetido en el tiempo. El bully o victimario siempre tiene la intención de dañar, mientras que la víctima generalmente presenta síntomas de angustia y depresión (Pepler y Craig, 2000); y en el peor de los casos, cuando no hay intervención por parte de los adultos y de los espectadores, el bully persigue continuamente a su víctima, la insulta y la descalifica hasta que logra que ésta se sienta humillada y desprestigiada. Sin importar la gravedad del bullying (incipiente o grave) resulta perjudicial para todos los involucrados y para la convivencia (Ortega, Del Rey y Elipe, 2012).

Generalmente, el bullying surge sin provocación alguna por parte de la víctima. Y cuando se da este caso, el bully siempre encuentra una excusa para justificar su comportamiento, y siempre atribuye a la víctima la culpa, 
ya sea porque tiene un defecto físico, un hábito o una actitud desagradable para éste. Por otra parte, Olweus (1993, citado por Losey, 2009) describe dos tipos de víctimas: la "pasiva" y la "provocativa". Limber et al. (2004, citados por Losey, 2009) afirman que la víctima pasiva tiende a ser tranquilo, tímido, cauteloso, sensible, inseguro, y tiene poca confianza; además, tienden a ser niños o niñas que son físicamente más débiles que sus pares.

\section{Convivencia en la escuela}

Para el Ministerio de Educación Nacional (MEN), la convivencia escolar:

Se puede entender como la acción de vivir en compañía de otras personas en el contexto escolar y de manera pacífica y armónica. Se refiere al conjunto de relaciones que ocurren entre las personas que hacen parte de la comunidad educativa, el cual debe enfocarse en el logro de los objetivos educativos y su desarrollo integral. (MEN, 2013, p.25)

Se deduce que la convivencia escolar, hace referencia a las relaciones interpersonales tolerantes y respetuosas que se dan entre los miembros de la comunidad educativa, lo cual permite que el clima escolar sea el adecuado, y que los procesos de enseñanza aprendizaje sean exitosos. En este sentido, Aragón-Jiménez (1999) afirma que la convivencia permite un desarrollo normal y saludable, ya que gracias a ella el ser humano ensaya importantes habilidades cognitivas, lingüísticas y socio-emocionales.

Por otra parte, para la Junta de Extremadura (2007), la convivencia va más allá de coexistir con los otros, de tolerarlos, implica aceptar las normas y el respeto mutuo de las ideas de otros y de sus estilos de vida, así como resolver de manera pacífica las tensiones producidas en la interacción. Es por ello, que las relaciones de convivencia deben sustentarse en el autoconocimiento de la propia manera de ser, pensar y sentir, en acciones personales coherentes, en la capacidad cognitiva para reflexionar sobre los conflictos teniendo en cuenta los valores universales, en la capacidad de diálogo, empatía y perspectiva social basada en valores como la cooperación y la solidaridad, en la competencia social que facilite la coherencia entre los criterios personales y las normas y principios sociales y en la resolución pacífica de conflictos (Junta de Extremadura, 2007, p. 40)

Lo anterior no implica que todo sea perfecto, y que nunca haya diferencias entre estudiantes, entre docentes y estudiantes etc., al contrario, como seres únicos y diferentes es natural que haya diferencias en la forma de pensar, de expresarse, de relacionarse, de ser y de vivir la vida. Por tanto es lógico que existan desacuerdos, pero esos desacuerdos representan oportunidades para educar en la paz, en la solución del conflicto y en el respeto por la diferencia y la diversidad.

Estos procesos de aprendizaje deben tener en cuenta que la convivencia no implica ausencia de conflictos. En todo grupo social se presentan este tipo de situaciones debido a la presencia de diversos puntos de vista y múltiples intereses; diferencias que pueden convertirse en motores de trasformación y cambio. (MEN, 2013, p.25) 
Al respecto, la Junta de Extremadura (2007), considera que para que las instituciones educativas sean espacios de aprendizaje de la convivencia es necesario que garanticen un clima escolar adecuado, en el cual los estudiantes, profesores, directivos y las familias puedan ejercer de forma armónica sus derechos y deberes (p.41).

\section{Implicaciones pedagógicas}

De acuerdo con, Ortega, Del Rey y Edipe (2012), las siguientes sugerencias contribuyen a prevenir el fenómeno del bullying escolar:

- Los sistemas educativos deben formar bien a los docentes, disponer de suficientes escuelas para todos, tener currículos comprensivos y en especial tienen que pensar que la convivencia diaria es fuente de bienestar y progreso.

- Es necesario que las escuelas entiendan que si su sistema de convivencia está basado en valores democráticos $\mathrm{y}$ respeto mutuo, será una organización eficiente y capaz.

- Una buena convivencia es un factor de protección contra el bullying.

- La escuela debe pensar en la distribución de poder y responsabilidad, la claridad y coherencia de las normas de disciplina, dada su incidencia en la convivencia.

- La escuela como sistema se debe posicionar en actitudes de rechazo a todo comportamiento de exclusión social. La mayoría de escolares conocen bien el funcionamien- to del bullying y son conscientes de la inmoralidad de la conducta implicada, pero no se traduce en sentimientos de oposición ante las conductas del agresor, ni en apoyo hacia la víctima.

- La escuela no debería permitir que una agresión pase de ser un intento de dominio o intimidación, que puede llegar a ser bien resuelto por la víctima y que el agresor saque la lección moral que le corresponde porque es responsable de lecciones de comportamiento y criterio moral.

- La disciplina poco clara e inconsistente, la incoherencia y división entre el profesorado y con el equipo directivo son factores de riesgo para la aparición de problemas de violencia.

- Los niveles de victimización de los propios docentes son fuente de facilitación de violencia en los estudiantes.

- Los lugares no supervisados por adultos como los pasillos, la escasa participación del alumnado en la dinámica de la escuela, la ausencia de procesos de detección y diagnóstico, la carencia de actividades de ocio y tiempo libre que estimulen el sentido de pertenencia y la falta de dinamización de los espacios de recreo, son variables que facilitan la aparición de problemas de violencia.

- La intervención educativa en red de iguales (pares), tiene una tendencia a ser la más efectiva a la hora de prevenir el fenómeno del bullying. 


\section{Metodología}

El estudio se desarrolló bajo el enfoque de Método Mixto. Según ValenzuelaGonzález y Flores-Fahara (2012), los métodos mixtos se caracterizan por combinar los enfoques cualitativo y cuantitativo para describir y explicar fenómenos en un solo estudio. Dicha combinación se puede realizar a través de la triangulación y la complementariedad pues permite corroborar los resultados obtenidos aplicando otros instrumentos, o a otras personas, así mismo con un método se pueden medir aspectos que el otro método no mide (Burke y Onwuegbuzie, 2004). Por otra parte Creswell y Plano (2007), señalan la existencia de varios tipos de estudios mixtos. El diseño paralelo convergente es uno de éstos estudios y se caracteriza por dar igual prioridad a los métodos cualitativos y cuantitativos durante todas las fases del proceso investigativo; cada método se desarrolla de manera independiente y durante el análisis y se mezclan los resultados lo que permite una interpretación global de la problemática; tal cual como se hizo en el presente trabajo.

A nivel cuantitativo, se implementó un estudio exploratorio tipo encuesta. La investigación por encuesta, incluye estudios que utilizan como instrumento de recogida de datos el cuestionario; son útiles para la descripción de un fenómeno educativo y para realizar una aproximación a la realidad o para estudios exploratorios (Bisquerra-Alzina, 2012, p. 233). Para Hernández-Sampieri, Fernández-Collado y BaptistaLucio (2003), los estudios exploratorios tienen como objetivo examinar un tema poco estudiado o que no ha sido abordado antes.
A nivel cualitativo, se implementó el estudio de caso. Para BisquerraAlzina (2012), los estudios de caso son estudios descriptivos, que aportan informe detallado del caso y no consideran hipótesis previas puesto que se realizan con el fin de aportar información sobre programas y prácticas sociales (p. 314).

\section{Participantes}

La muestra la conformaron estudiantes de grado quinto, los profesores directores del grupo de los respectivos estudiantes y directivos (orientadores escolares y coordinadores de convivencia) de una institución educativa de carácter público, ubicada en un entorno de violencia de la ciudad de Bogotá, localizada en la Localidad de Suba Rincón.

\section{Instrumentos}

Para cumplir con los objetivos propuestos, se diseñaron y validaron tres instrumentos de investigación con el fin de triangular la información. Dichos instrumentos fueron: una entrevista semi estructurada de carácter cualitativa para docentes y directivos, dos encuestas de carácter cuantitativo: una para determinar la percepción de convivencia en la institución, y una encuesta de percepción de convivencia y bullying en el aula.

Para la validación se realizó una prueba piloto y se contó con el juicio de un experto. Para procesar y facilitar el análisis de los datos cualitativos se utilizó el programa ATLAS.ti (versión 7) y para los datos cuantitativos se empleó el programa IBM SPSS. Statistics 24.0 


\section{Resultados}

\section{Percepciones de convivencia en la institución educativa}

Una de las unidades de análisis tenidas en cuenta para la investigación fue la agresión escolar y sus manifestaciones en el aula.

$\mathrm{Al}$ encuestar a los estudiantes del grado quinto de básica primaria, sobre su percepción sobre la convivencia al interior de la institución escolar, se encontró que el insulto es la forma de violencia más utilizada por los estudiantes dentro del colegio, con un $74 \%$. En menor medida, los estudiantes perciben que atemorizar al otro es otra manera de agresión que afecta la convivencia, en un 35\%, la evasión de clases con un 29\%; y las peleas con un $19 \%$.

\section{Formas de violencia escolar en la institución}

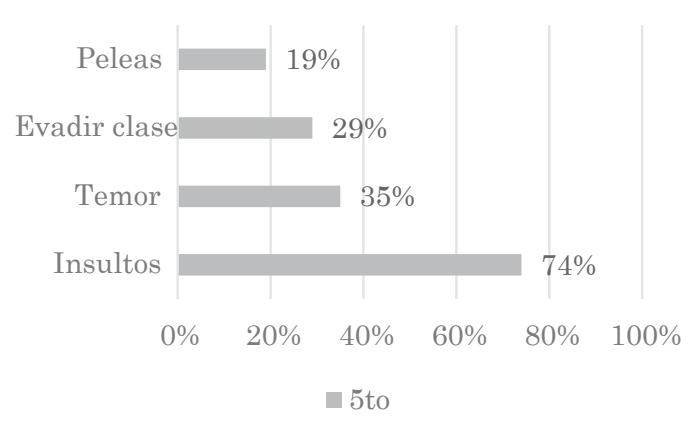

Fig. 1. Formas de violencia escolar en la institución Fuente: Elaboración propia.

De acuerdo con la entrevista realizada a un profesor del grado en cuestión, algunas de las razones por las que un $74 \%$ de los estudiantes de $5^{\circ}$, tienen la percepción del insulto como la manera de agresión más frecuen- te son: cuando los padres de familia son citados para hablar de un determinado comportamiento de sus hijos, lo que se observa es que delante del profesor o directivos los corrigen a través de palabras soeces; de hecho los estudiantes manifiestan que en el hogar el trato verbal no es respetuoso, por el contrario es "humillativo" y grosero; por lo que se infiere trasladan la manera habitual en que son tratados en el contexto familiar a las relaciones interpersonales en contexto escolar.

Referente al resultado de sentir temor, el cual se presenta en esta institución con una frecuencia de $35 \%$, explica un profesor entrevistado que es algo que sienten muchos pero no lo expresan abiertamente por la misma condición de sentir miedo ante la amenaza.

En referencia a la evasión de clases, el $29 \%$ de los estudiantes lo percibe como una práctica frecuente y común en esta institución. Docentes entrevistados explican que influye el que algunos estudiantes de primaria tienen primos y/o hermanos en grados superiores que los incentivan a adoptar este comportamiento ante el aburrimiento académico, por no asumir responsabilidades como el incumplimiento de los deberes y /o por preferir otras actividades que captan en mayor medida su atención. Adicionalmente influye también el que los salones de $5^{\circ}$ estén ubicados geográficamente cerca de los estudiantes de básica secundaria, lo que permite contacto con éstos, de quienes escuchan y aprenden todos los comportamientos que les genera interés ya que por su edad están en busca de su identidad. 
Aunque las peleas se identificaron con un menor porcentaje (19\%) de percepción, estas se manifiestan en la cotidianeidad escolar y según explicaciones de docentes y coordinadores de convivencia están directamente relacionadas con la edad y el ciclo vital de esta población estudiantil. El origen de estas peleas se relacionan con situaciones como: "me quitó el novio o novia, me miró mal, pasó y me alcanzó a tocar, me dijo que esta fea, que otra compañera era más bonita, etc".

Ortega, Del Rey y Elipe (2012) afirman que el conflicto puede tener como finalidad en el ser humano reforzar la identidad y enriquecer la competencia social. Una de sus funciones, es que los individuos aprendan que los problemas sociales sirven para conocerse a sí mismo, especialmente a nivel afectivo, para saber cuándo cambiar o retirar los sentimientos hacia alguien, quiénes o quién realmente puede ser un amigo o amiga, y en palabras sirven para "recomponer las redes afectivas" ( $p$. 208).

\section{Percepciones de convivencia al interior del aula}

Los estudiantes perciben que al igual que en la institución, en el aula el insulto es la forma de agresión más frecuente (58\%), seguido de las burlas y la agresión física con un 52\%. Por último, el $48 \%$ percibe el uso de apodos como agresión. Insultos, burlas y agresión física se abordarán con mayor detalle en el siguiente apartado.

\section{Formas de violencia escolar en el aula de clase}

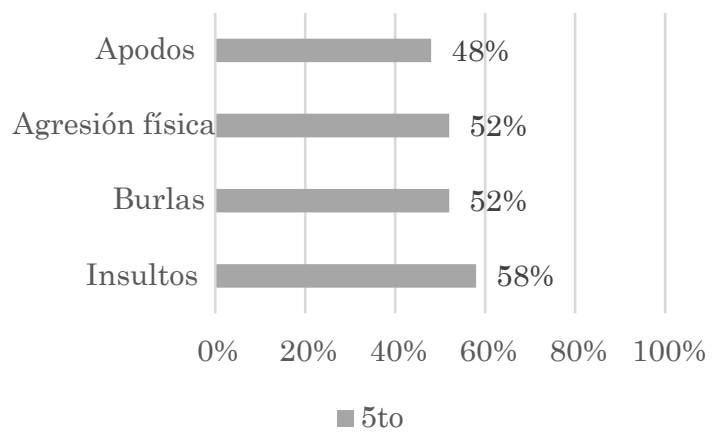

Fig. 2. Formas de violencia escolar en el aula de clase Fuente: Elaboración propia.

En cuanto a los apodos, es una práctica común entre los estudiantes, utilizada como forma de agresión. Profesores y coordinadores entrevistados afirman que en ocasiones son los mismos docentes quienes alientan esa conducta en los estudiantes, porque usan el apodo para ridiculizarlos ante sus compañeros, comportamiento que los estudiantes aprenden, imitan y utilizan en la convivencia escolar. En la escogencia del apodo hay gran creatividad e intencionalidad de molestar a la otra persona, utilizando una de sus características particulares, es así como que a quien usa gafas le digan "cuatro ojos", o "el ciego", a quien tiene baja estatura: "el enano" o "pigmeo", a quien tiene buen desempeño académico, "el nerd", a quien reconoce verbalmente una falta cometida, se le apoda "el sapo", a quien tiene acné, le dicen "tarrito rojo", a quien tiene apellidos tales como Caro o Barato, lo apoden "regalado", etc. Se trata entonces de una forma de discriminación ya sea por características físicas, formas de ser, por el nombre o por el apellido, comportamentales, habilidades o bajos 
desempeños en algún campo. $\mathrm{Al}$ respecto plantea Mateos (2015) que:

Los sobrenombres/apodos es un núcleo de violencias escolares, dado que es en este ámbito donde se generan y circulan la mayor parte de los sobrenombres. Estos hacen clivaje fundamentalmente en discriminaciones por: aspecto físico, uso de anteojos, obesidad, fealdad/belleza, tartamudez, sordera, ceguera, etc. (p.12)

\section{Expresiones del Bullying en el aula}

Comúnmente se reconocen personas que intervienen en el bullying: el victimario y la víctima, pero es importante reconocer que hay un tercer implicado en el fenómeno y es el espectador.

\section{Acciones del victimario}

$\mathrm{Al}$ cuestionar a los estudiantes, sobre las acciones que como victimarios suelen realizar dio como resultado que la burla es el primer mecanismo de agresión que utilizan, con un $42 \%$, seguido a este se tiene el insulto con un $35 \%$ y por último, la agresión física con un $23 \%$.

\section{Acciones como victimarios}

o agresores

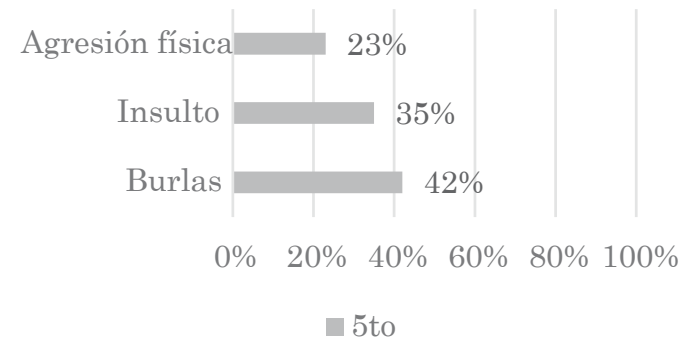

Fig. 3. Acciones como victimarios o agresores Fuente: Elaboración propia.
Ahora bien, la burla es un mecanismo de agresión fuerte en el aula de $5^{\circ}$ de primaria, con un $42 \%$, siendo este el porcentaje más alto dentro de los ítems analizados. En la entrevista realizada a un profesor sobre este tema, y en específico cuando se le pregunta por la razones que hacen que esta sea el mecanismo más utilizado por los agresores, manifiesta que a pesar de que esta situación se encuentra tipificada en el manual de convivencia, los estudiantes agresores, no ven este acto tan trascendental y por consiguiente la sanción que este tipo de actos conlleva no representa gran peligro para ellos, de tal manera que lo siguen haciendo. Por ejemplo, cuando se llaman a las partes a conciliar (víctima y victimario), se observa como el estudiante quien es el agresor acepta rápidamente su culpa y de inmediato dice "perdón, o disculpas" y eso es todo, de ahí no pasa. Lo más impactante es ver cómo a los pocos minutos de haber ofrecido disculpas, el agresor vuelve a proceder de la misma manera o peor.

Con menor frecuencia se percibe la presencia de insultos (35\%) y agresión física (23\%), los cuales responden a comportamientos aprendidos tanto del contexto familiar como el social (vecindario barrial), al punto que se incorpora que esta sea la forma de resolución de conflictos y no las basadas en el diálogo y el respeto por la diferencia.

La naturaleza del bullying y de la victimización cambia con la edad y se diferencia por sexo. Hay una tendencia de disminución del fenómeno de bullying a medida que avanzan los niños en la escuela, al igual que hay una 
reducción del uso de medios físicos en los grados superiores (Olweus, 1993, citado por Losey, 2009). Además, los niños muestran comportamientos de bullying con más frecuencia que las niñas (Kumpulainen, Rasanen, y Henttonen, 1999, citado por Losey, 2009). Los niños son más propensos a causar la agresión física directa con la intención de causar daño físico, mientras que las niñas tienen más probabilidades de causar formas indirectas de agresión con la intención de causar un daño psicológico. Sin embargo, no hay diferencias significativas de género en el bullying verbal directo (Baldry y Farrington, 1999, citado por Losey, 2009).

\section{Reacciones de la víctima}

Otro aspecto evaluado, fue la reacción de las víctimas frente a las agresiones recibidas por parte del victimario o agresor. Se encontró que el 55\% de los estudiantes agredidos guardan silencio. Al entrevistar al docente por esta situación, manifestó que una posible explicación es por el temor a denunciar. Es decir, cuando un estudiante agrede a otro, generalmente lo amenaza con causarle un daño mayor si llega a denunciarlo. También ocurre que el agresor amenaza con sacar del grupo al agredido, es decir dejarlo sin amigos, y es en ese momento en donde comienza una campaña de desprestigio hacia ese estudiante, hasta que quede solo. De tal manera que al saber el alcance de este agresor, ninguno quiere entonces retarlo ya que saben que de cualquier manera van a perder. La práctica del bullying requiere que la víctima posea características particu- lares, tales como que sean inseguros, ansiosos, tímidos, tranquilos, cautelosos, sensibles y con poca confianza en sí mismo, lo cual le facilita al victimario que se guarde silencio (Losey, 2009)

Otra reacción que se encontró es la de comunicarle al profesor con un $52 \%$. $\mathrm{Al}$ indagar sobre esta respuesta, el docente manifiesta que para algunos estudiantes la figura del profesor representa a la persona que puede proteger y que tiene autoridad.

Comunicarle a la familia es otra forma que tiene el agredido de reaccionar con un $42 \%$, esta forma de actuar es más baja y es dada por aquellos estudiantes quienes tienen unos padres pendientes y con tiempo suficiente para dedicarle a los asuntos escolares de sus hijos, logrando su confianza.

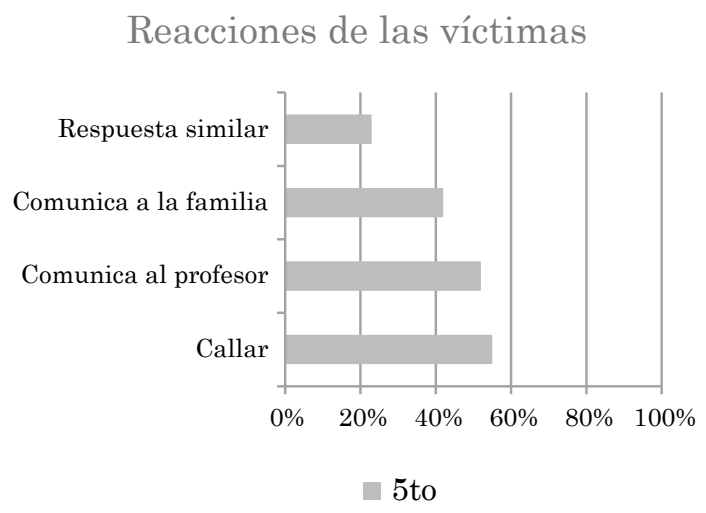

Fig. 4. Reacciones de las víctimas Fuente: Elaboración propia.

\section{Acciones de espectadores}

Otro aspecto que se tuvo en cuenta dentro del análisis es el comportamiento de los estudiantes quienes son los espectadores de las agresiones entre pares, se halló que el $84 \%$ interviene 
o se involucra haciendo uso del insulto, el $81 \%$ maltratando o golpeando; el $77 \%$ ignora el acto de agresión, y el 68\% se burla.

Frente a estos resultados, docentes y coordinadores entrevistados manifestaron que este tipo de reacciones depende del grado de amistad que se tenga con el agresor o el agredido, ya que si se es amigo del agresor, ayudará a la agresión. Pero si se es amigo del agredido hará lo que sea necesario para defender a su compañero (a).

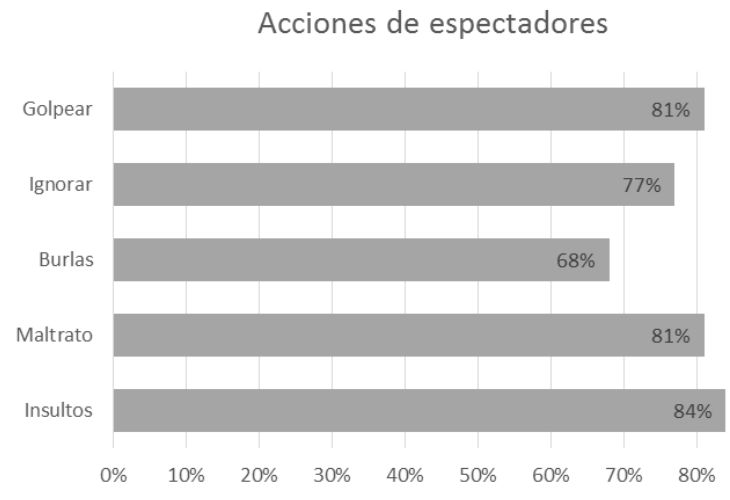

Fig. 5. Acciones de los espectadores Fuente: Elaboración propia.

El espectador hace parte de la dinámica del bullying y está involucrado con un determinado grado de participación y depende en cierta medida del grado de dominio del agresor, pues este se caracteriza por tener la habilidad de crear un círculo social que lo apoya, estimula, acepta o participa en la agresión. La mayoría de espectadores "conocen bien el funcionamiento del bullying y son conscientes de la inmoralidad de la conducta implicada, pero no se traduce en sentimientos de oposición ante las conductas del agresor, ni en apoyo hacia la víctima" (Ortega, Del Rey y Edipe, 2012, p. 224), así como se refleja en la gráfica anterior.

\section{Escenarios de violencia en la escuela}

$\mathrm{Al}$ ser encuestados los estudiantes de $5^{\mathrm{o}}$ sobre los escenarios de agresión en la escuela, se hizo evidente que en los sitios en los que frecuentemente se dan los actos de agresión son: con un $74 \%$ en los pasillos, seguido del patio con presencia del profesor con un $68 \%$, y fuera de la institución en un $65 \%$. Y en el sitio en donde se presentan agresiones pero en menor medida es el aula con o sin profesor.

Lo anterior deja ver que los agresores aprovechan el momento en el que su víctima está sin la presencia de alguien que lo defienda, es por esto que la agresión en los pasillos es mayor. Cuando se presenta la agresión en presencia de algún profesor, es porque este profesor no es el director de curso ni de ninguna asignatura que curse y no lo perciben como una figura de autoridad. Aunque es más baja la agresión fuera de la institución, se presenta ya que en este lugar saben que nadie va a hacer nada por detenerlos. Se trata de conflictos que se generan en la institución educativa pero que se resuelven en un lugar externo y acordado entre las partes involucradas.

Escenarios de violencia en la escuela

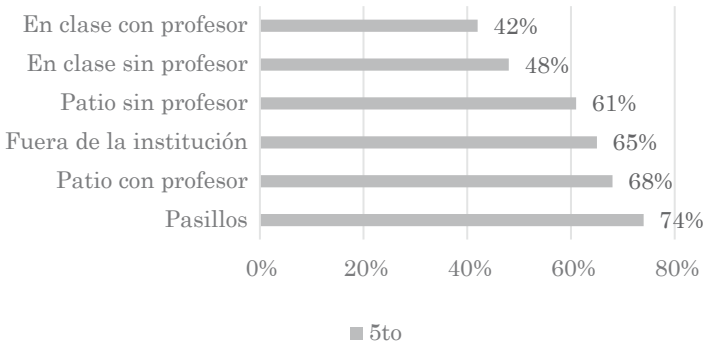

Fig. 6. Escenarios de violencia en la escuela Fuente: Elaboración propia. 


\section{Prácticas pedagógicas para la convivencia en el aula}

Para el grado $5^{\circ}$, según información suministrada por docentes y coordinadores de esta institución no existe un proyecto estructurado para fortalecer la convivencia escolar en este nivel poblacional, como si lo hay para los estudiantes de educación básica secundaria. Las prácticas existentes se limitan al trabajo del área académica y sus procesos de enseñanza - aprendizaje dejando de lado la parte valorativa, aspecto fundamental en la formación integral de los niños y niñas, lo que permite entrever que pese a las normatividades establecidas en torno a problemáticas escolares, las acciones pedagógicas por parte de la comunidad educativa no toman parte al momento de mediar o solucionar un conflicto, pues solo se lleva a cabo lo establecido legalmente por el Ministerio de Educación Nacional MEN o los lineamentos dados por la Secretaria de Educación Distrital SED.

\section{Conclusiones}

El estudio permitió concluir los siguientes aspectos centrales, que se profundizan a continuación en relación con las percepciones de Bullying y Convivencia Escolar. La institución educativa no reconoce ni valora la importancia de la formación para la convivencia y le da prioridad al trabajo relacionado con el aprendizaje de conceptos y la transmisión de conocimientos, lo cual repercute en crear un ambiente en el que el conflicto se resuelve mediante agresión y violencia y no hay espacio para el diálogo, ni para el respeto al otro y a la diferencia.
En esta misma línea los niños, niñas y jóvenes aprenden la valoración dada, tanto por la institución como por la familia, al aspecto convivencial y lo expresa en su comportamiento cotidiano en la relación con sus compañeros de aula.

Algunos docentes reconocieron la carencia de formación ante el manejo del conflicto y la prevención del bullying y en ocasiones hasta son ellos mismos quienes propician comportamientos violentos en la institución educativa. Se reproduce así una especie de ciclo de violencia, en donde el docente aplica lo que vivió en su infancia. Es así como Cardozo (2010) reconoce la necesidad de trabajar más en la formación en convivencia como una posibilidad de generar procesos reflexivos y hacer posible convivir con el otro, aspectos indispensables para promover procesos de multiplicación hacia las comunidades, colegios y familias, respondiendo así a la necesidad de formar a otras personas en relación a la convivencia, como ejercicio permanente de construcción social. Este planteamiento converge con los aportes de Avendaño-Villa, Cortés-Peña y Guerrero-Cuentas (2016) en relación con la importancia que tienen los procesos asociados al desarrollo de las competencias sociales en estudiantes dentro de los contextos escolares y familiares vulnerables.

Se vio la necesidad de que en las Instituciones educativas se formulen y apliquen programas pedagógicos enfocados a prevenir el manejo violento del conflicto y a formar en la consolidación de una cultura de convivencia. Al respecto reconoce Delors (1994), que esta labor educativa debe iniciarse desde los primeros años de vida de la 
persona y que debe ser una práctica escolar cotidiana, que cuente con la participación de los profesores y estudiantes en el desarrollo de proyectos comunes que permitan el aprendizaje de estrategias para el manejo de conflictos y sean una referencia para la vida futura de estos infantes. En tal sentido, se evidencia la necesidad de seguir avanzando en el diseño de modelos que permitan comprender estructuralmente los factores asociados con la prevención de la violencia escolar y el desarrollo de las conductas prosociales en el marco de la convivencia escolar (Valderrama, Peña y Rodríguez, 2001).

A pesar de que el Estado expidió la Ley 1620, conocida como Ley de convivencia y que además incluye unas preguntas relativas a convivencia y competencias ciudadanas, en las pruebas realizadas a estudiantes de educación media, no va más allá de lo normativo ni da orientaciones a las instituciones educativas. En este sentido expresan Salas y Cómbita (2017) que la escuela refleja la dinámica social, y que mientras el proyecto de nación considerado en la constitución política y en sus leyes, pero que no trasciendan de lo normativo y se incentive el ejercicio de buenas prácticas relacionadas con convivencia y paz, "la escuela seguirá siendo un lugar que refleje una sociedad disfuncional marcada por la solución de los conflictos por la vía violenta". Agregan además estos autores, que las políticas públicas generalmente fracasan puesto que no están alineadas con la conducta natural de las personas, debido precisamente a la falta de entendimiento del comportamiento humano (Salas y Cómbita, 2017, p. 68).
Delors (1994), reconoce que si la relación interpersonal se establece en un contexto de igualdad y se formulan objetivos y proyectos comunes, los prejuicios y la hostilidad subyacente pueden dar lugar a una cooperación más serena e, incluso, a la amistad. También afirma que la educación tiene una doble misión: enseñar la diversidad de la especie humana y contribuir a una toma de coincidencia de las semejanzas y la interdependencia entre todos los seres humanos. Desde la primera infancia, la escuela debe aprovechar todas las oportunidades que se presenten para esa doble enseñanza. Este cambio implica el trabajo comprometido de todos los miembros de una comunidad académica, como única posibilidad de lograr al aprendizaje requerido para una convivencia asertiva. Al respecto Delors (1994) concluye que el enfrentamiento, mediante el diálogo y el intercambio de argumentos, serán unos de los aprendizajes fundamentales de la educación del siglo XXI.

\section{Referencias}

ACNUR. (2010). Directrices de elegibilidad del ACNUR para la evaluación de las necesidades de protección internacional de los solicitantes de asilo de Colombia. Alto Comisionado de las Naciones Unidas para los Refugiados (ACNUR). Recuperado de: http://www.acnur.org/t3/filead$\mathrm{min} / \mathrm{script} / \mathrm{doc} . \mathrm{php}$ ?file=t3/fileadmin/Documentos/BDL/2010/8118

Aragón-Jiménez, E. (1999). Educar para la convivencia. (Proyecto de Investigación Educativa subvencionado por la Consejería de Educación y Ciencia de la Junta de Andalucía). España 
Avendaño-Villa, I., Cortés-Peña, O. F., \& Guerrero-Cuentas, H. (2015). Competencias sociales y tecnologías de la información y la comunicación como factores asociados al desempeño en estudiantes de básica primaria con experiencia de desplazamiento forzado. Diversitas, 11(1), 13-36.

Bisquerra- Alzina, R. (2012). Metodología de la investigación educativa. Madrid: La Muralla.

Bogotá. (19 de enero de 2015). Bogotá perdió el año en seguridad: mayoría de delitos aumentaron. El Tiempo, Sección Bogotá. Recuperado de: http://www.eltiempo.com/bogota/ inseguridad-en-bogota-aumentanasesinatos-rinas-y-robos/15115085

Burke, J. y Onwuegbuzie, A. J. (2004). Mixed methods research: A research paradigm whose time has come. Educational Research, 33, 714-726.

Cardozo A (2010) La formación para la convivencia: Un reto de la contemporaneidad. CUC Barranquilla, Colombia.

Creswell, J. W. y Plano, V. (2007). Designing and conducting mixed methods research. California: SAGE Publications.

Delors, Jacques (1994). "Los cuatro pilares de la educación", en La Educación encierra un tesoro. México: El Correo de la UNESCO, pp. 91-103.

Guerrero-Barón, J., y García-Sánchez, B. Y. (2012). Violencias en contexto. La ciudad, el barrio y la violencia escolar. Bogotá, Colombia: Universidad Distrital Francisco José de Caldas.

Hernández-Sampieri, R., Fernández. Collado, C. \& Baptista-Lucio, P. (2003). Metodología de la investigación. México: McGraw-Hill.
Junta de Extremadura. (2007). Plan regional de la convivencia escolar en Extremadura. Extremadura, España: Junta de Educación.

Ley 1620 de 2013 - Decreto 1965 de 2013

Ministerio de Educación Nacional. (2013). Guías pedagógicas para la convivencia escolar. Ley 1620 de 2013 - Decreto 1965 de 2013. Guía 49. Bogotá, Colombia: Amado Impresores.

Mateos, P. (2015). Narrativas de la violencia. Buenos Aires: CLACSO.

Ortega, R., Del Rey, R., y Elipe, P. (2012). Violencia escolar y bullying: El estado de la cuestión y los nuevos retos investigadores. En, Reflexiones sobre la violencia en las escuelas. (pp. 205230). México, D.F.: Siglo XXI Editores

Pinheiro, P. S. (2006). Informe mundial sobre la violencia contra los niños y niñas. Estudio del Secretario General de las Naciones Unidas sobre la Violencia contra los Niños. Nueva York: Publicación de las Naciones Unidas.

Salas, K. y Combita, H. (2017). Análisis de la convivencia escolar desde la perspectiva psicológica, legal y pedagógica en Colombia. Cultura. Educación y Sociedad 8 (2), 7992.

Secretaría de Educación Distrital, Secretaría Distrital de Cultura, Recreación y Deporte, Secretaría Distrital de la Mujer y la Universidad Distrital Francisco José de Caldas. (2013). Encuesta de clima escolar y victimización 2013. Bogotá, Colombia: Universidad Distrital Francisco José de Caldas. 
Unicef. (2006). La violencia contra niños, niñas y adolescentes. Informe de América Latina en el marco del Estudio Mundial de las Naciones Unidas. Recuperado de http://www. unicef.org/lac/Informe_Mundial_ Sobre_Violencia_1(1).pdf

Valderrama, B. P., Peña, O. F., \& Rodríguez, D. E. (2001). Aplicación del modelo de ecuaciones estructurales en la explicación del comportamiento social infantil y juvenil en el estrato socio-económico bajo la ciudad de Bogotá. Suma Psicológica, 8(1), 1-54.
Valenzuela-González, J. R. y FloresFahara, M. (2012). Fundamentos de Investigación Educativa (2). Monterrey, Nuevo León, México: Editorial Digital del Tecnológico de Monterrey. 